\title{
Dunkhase, Jan Eike (Hrsg.): Reinhart Koselleck, Carl Schmitt. Der Briefwechsel. 1953-1983, 459 S., Suhrkamp, Berlin 2019.
}

\section{Christof Dipper}

Online publiziert: 12. Mai 2020

(C) Der/die Autor(en) 2020

„So wurde, wer sich bei Carl Schmitt bedankt, zum Sprachrohr von Carl Schmitt abgestempelt". Reinhart Koselleck beklagte sich mit diesen Worten noch 2004, bei der Feier zur 50. Wiederkehr seiner Promotion, über den Schatten, der auf „Kritik und Krise" nun schon ein ganzes Gelehrtenleben lang laste. Ob zu Recht, ist bis heute umstritten. Jedenfalls ist das Verhältnis Kosellecks zu Schmitt Gegenstand ungezählter wissenschaftlicher Arbeiten. Die allermeisten von ihnen beschränken sich auf eine Kombination von Textphilologie und Ideengeschichte, bewährten Verfahren sicherlich, aber ihre Aussagen standen bisher fast immer unter dem Vorbehalt, dass sie nicht am Material der Nachlässe verifizierbar waren. Nun liegt der Briefwechsel der beiden Protagonisten in mustergültiger Edition vor und die Hoffnung, endlich herauszufinden, ,wie es eigentlich gewesen', ist naturgemäß groß.

Hat Schmitt also Koselleck beeinflusst, vor allem natürlich bei seiner Dissertation, lautet die Gretchenfrage? Und die Antwort heißt: Ja, aber anders als in der Regel angenommen.

Zunächst ein paar allgemeine Bemerkungen zum Briefwechsel: Er umfasst in der Zählung des Herausgebers Jan Eike Dunkhase 119 Briefe, jedoch belaufen sich alle Sendungen zusammen auf 141 Stück (plus zwei an Kosellecks Frau). Die Mehrzahl davon, nämlich 74, stammt aus der Hand Kosellecks. Etliche sind nur Widmungen. Zieht man diese ab, bleiben nur 87 , echte ‘ Briefe. Andere Briefwechsel Schmitts sind umfangreicher, der mit seinem Schüler Ernst Forsthoff etwa kommt auf eine Anzahl von 358; dort ist auch der Tonfall oft sehr anders, beide lassen ihren Animositäten freien Lauf. Koselleck dagegen wechselte mit niemand anderem auch nur annähernd so viele Briefe. Diese verteilen sich nicht gleichmäßig auf die 30 Jahre. Die mit Abstand größte Dichte, nämlich 16 Briefe, betrifft das Doppeljahr 1953/54, als Koselleck seine Dissertation schrieb. Danach pendelt sich die Zahl auf jährlich zwei bis drei Briefe ein (1964 und 1982 allerdings gar keine, dagegen 1979 sechs). Sie sind auch unterschiedlich lang, je älter Schmitt wird, desto kürzer werden seine Nachrichten; das Schreiben wird zur Last.

Dunkhase überschreibt sein Nachwort mit „Asymmetrische Korrespondenz“. Das ist sie in der Tat, und zwar in mehr als einer Hinsicht. Neben der numerischen gibt

C. Dipper (ه)

Technische Universität Darmstadt, Darmstadt, Deutschland

E-Mail: christof.dipper@tu-darmstadt.de 
es auch eine protokollarische Asymmetrie - Koselleck schreibt stets an den „sehr verehrten Herrn Professor“ und unterzeichnet vielfach als „sehr ergebener“, während Schmitt sich zunehmend jovial gibt (,Ihr alter Carl Schmitt“) - und dann vor allem eine inhaltliche. Koselleck zeigt sich lebenslang als dankbarer und deshalb gelegentlich bis zur Beflissenheit hilfsbereiter Schüler, ignoriert alle Zumutungen der Schmittschen Selbstinszenierung und übergeht auch so gut wie alle Klagen Schmitts über sein Schicksal als die Unperson der frühen Bundesrepublik. Vor allem aber und das ist vielleicht die größte Überraschung dieses Briefwechsels - sticht die intellektuelle Asymmetrie der Briefe von Anfang ins Auge. Aus Plettenberg zurückkommend, skizziert der knapp dreißigjährige Doktorand Koselleck schon in seinem ersten Brief vom 21. Januar 1953 einen geistigen Horizont, der wahrlich staunen macht. Er entfaltet hier eine aus Versatzstücken der Konservativen Revolution und der antihistoristischen Perspektive auf die Vergangenheit zusammengesetzte Kritik der Historiographie, die er mit einer begriffsanalytisch unterstützten Kampfansage an jegliche Geschichtsphilosophie verkoppelt und aus diesen Argumenten ein Programm entwickelt, von dem er sich eine Entlarvung der sich als fortschrittlich und vernünftig ausgebenden Utopien und damit ,eine Antwort auf unsere konkrete Situation" erhofft (S. 11).

In der Tat war damals der Beitrag der Geschichtswissenschaft zur Gegenwartsdeutung dürftig. Nicht von ungefähr nennt Koselleck lediglich Hans Freyers „,Weltgeschichte Europas“ von 1948 als für seine Fragen hilfreich, während er mit Friedrich Meinecke, Karl Jaspers, Alexander Rüstow und eigentlich auch mit Martin Heidegger nichts anfangen konnte, weil diese sich über ,die stillschweigenden Voraussetzungen der Geschichtsphilosophien“ (S. 10) nicht im Klaren seien. Bekanntlich war Koselleck die antiquarische Geschichtswissenschaft ein Leben lang fremd; stattdessen versuchte er durch theoretisch gebotene, oft weit nach rückwärts geschlagene Schneisen das Heute zu verstehen.

Carl Schmitts Antwort auf dieses intellektuelle Brillantfeuerwerk fiel bemerkenswert schwach aus. Er forderte Koselleck zum Weitermachen auf und dazu, ,dem Geschwafel“ sein ,einfaches, dreiteiliges, festes Bild entgegen[zusetzen]“. Schließlich fügte er einen Satz hinzu, der nicht weniger ,Geschwafel“ ist: „Die Redlichkeit in Hinsicht auf den dauernden Ursprung der Geschichte, das ist es“ (S. 17).

Es ist diese Asymmetrie, die den Briefwechsel der beiden lebenslang kennzeichnet und die Antwort auf die Gretchenfrage liefert. Schmitts Funktion für Koselleck war, von seiner „strengen Mahnung“ (S. 9) zur semantischen Kontrolle abgesehen, eine „mäeutische“, wie Dunkhase es nennt (S. 419). Sein geschicktes Fragen provozierte - nicht nur Koselleck - zur Formulierung von Gedanken, die schon im Kopfe schlummerten, aber erst durch das Gespräch, sei es in Heidelberger Kneipen, sei es in Plettenberg, ihren Weg aufs Papier fanden. Im gesamten Briefwechsel bedankt sich Koselleck immer wieder für die Anregungen, die er bei seinen Besuchen in Plettenberg erhalten habe (die mäeutische Funktion Schmitts spricht er direkt in Brief Nr. 20 an), es finden sich aber keine Stellen, in denen er Schmitt jemals konkret um Rat fragte oder mit ihm eine neu angegangene Frage diskutierte. Schmitt hatte nach 1945 das biografisch bedingte Verlangen und offenkundig auch die Gabe, vielversprechende junge Menschen zu entdecken und an sich zu binden, aber der über Sechzigjährige bot diesen - soweit sie keine Juristen waren - inhaltlich außer 
seinem alternativen Blick auf die pathologische Moderne (nicht Martin Luther oder Otto von Bismarck waren ,die Bösen“, sondern die Aufklärer) keine nennenswerten Anregungen. Die substanziellen Aussagen zur Zeitschwelle um 1800 stammen nicht von Schmitt, bei ihm fanden sich nur ressentimentgeladene, doch genügte das intelligenten Lesern wie Koselleck, Hanno Kesting, Christian Meier, Nicolaus Sombart und anderen, um daraus höchst produktive Ideen abzuleiten.

Die Briefedition fördert auch Verwunderliches zutage, so zum Beispiel, wie die beiden eine positive Besprechung von ,Kritik und Krise“ an die Öffentlichkeit brachten. Nachdem Schmitt vom „Historisch-Politischen Buch“ um eine Rezension gebeten wurde, teilte er das seinem Schützling sogleich mit und bot ihm an, aus zwei Versionen eine auszuwählen. Koselleck nahm das Angebot nach ausgeräumten Bedenken an und entschied sich verständlicherweise für diejenige, die nicht Schmitts Neigung zur Selbstbezüglichkeit, sondern sein eigenes Vorhaben wiedergab (beide Versionen sind im Anhang abgedruckt).

Es wäre noch viel Interessantes aus diesem wunderbaren Quellenbestand zu berichten, doch ist hier nicht der Platz dafür. Daher nur so viel: Die allmähliche Entfernung Kosellecks von den Denk- und Argumentationsfiguren Schmitts findet im Briefwechsel höchstens insofern ein Echo, als letzterer auf die ihm übersandten Arbeiten je später, desto weniger mit sachlichen Argumenten eingeht. Dennoch inszeniert sich Koselleck in seinen Briefen bis zum Schluss als dankbarer ,Schüler", der seinen ,Lehrer“ seit 1977 mehrfach drängt, ,eine solche Auswahl Ihrer Schriften herauszugeben, die vergleichsweise konstante Probleme unseres Jahrhunderts (als Geschehenseinheit) thematisieren“ (S. 321). Damit deutet sich zugleich eine Kontinuität Kosellecks in seiner - radikal pessimistischen - Zeitdiagnose an. Tatsächlich schreibt er Schmitt im folgenden Jahr: „So bleiben die alten Fragen aktuell und wohl dauerhaft unlösbar, Fragen, die ich im Gespräch mit Ihnen nach dem Kriege zu stellen begonnen hatte [...]. Ich wiederhole sie heute, um Ihnen - aus den Ferien - ein Zeichen meines Dankes zu senden für die jahrzehntelangen Anregungen, die meinen wissenschaftlichen Lebenslauf begleitet haben“" (S. $331 \mathrm{f}$.). Koselleck scheint den seit seiner Dissertation erheblich gewandelten politischen Rahmenbedingungen keine Rechnung zu tragen. Es sieht fast so aus, als seien ausgerechnet bei ihm persönlich Erfahrungsraum und Erwartungshorizont nicht auseinandergetreten.

Funding Open Access funding provided by Projekt DEAL.

Open Access Dieser Artikel wird unter der Creative Commons Namensnennung 4.0 International Lizenz veröffentlicht, welche die Nutzung, Vervielfältigung, Bearbeitung, Verbreitung und Wiedergabe in jeglichem Medium und Format erlaubt, sofern Sie den/die ursprünglichen Autor(en) und die Quelle ordnungsgemäß nennen, einen Link zur Creative Commons Lizenz beifügen und angeben, ob Änderungen vorgenommen wurden.

Die in diesem Artikel enthaltenen Bilder und sonstiges Drittmaterial unterliegen ebenfalls der genannten Creative Commons Lizenz, sofern sich aus der Abbildungslegende nichts anderes ergibt. Sofern das betreffende Material nicht unter der genannten Creative Commons Lizenz steht und die betreffende Handlung nicht nach gesetzlichen Vorschriften erlaubt ist, ist für die oben aufgeführten Weiterverwendungen des Materials die Einwilligung des jeweiligen Rechteinhabers einzuholen.

Weitere Details zur Lizenz entnehmen Sie bitte der Lizenzinformation auf http://creativecommons.org/ licenses/by/4.0/deed.de. 